\title{
Phosphorylated Signal Transducer and Activator of Transcription 1 is a potential predictor of interferon response in patients with advanced renal cell carcinoma
}

\author{
WEI LI ${ }^{1}$, QIANG WEI ${ }^{2}$ and JIANBO LIANG ${ }^{1}$ \\ Departments of ${ }^{1}$ Urology and ${ }^{2}$ Pathology, The People's Hospital of Guangxi Zhuang Autonomous Region, \\ Nanning, Guangxi 530021, P.R. China
}

Received August 22, 2013; Accepted February 22, 2014

DOI: $10.3892 / \mathrm{mmr} .2014 .2046$

\begin{abstract}
The present study aimed to evaluate the expression status of Janus kinase (JAK)-Signal Transducer and Activator of Transcription (STAT) in renal cell carcinoma and benign renal tissue, and identify a potential biomarker for interferon (IFN) response prediction. A total of 32 specimens of human renal cell carcinoma and 10 specimens of benign renal tissue were harvested from surgically removed kidneys. The expression levels of JAK-STAT were determined by immunohistochemical staining and quantitative polymerase chain reaction. Furthermore, the expression levels of JAK-STAT in renal cell carcinoma tissues that were stimulated with IFN- $\alpha$ were quantified by western blot analysis. The positive expression rates of JAK1, STAT1 and phosphorylated (P)-STAT1 in the renal cell carcinomas were significantly lower than that in the benign renal tissues $(25.0,31.2$, and $12.5 \%$ vs. $70.0,50.0$, and $70.0 \%$, respectively; $\mathrm{P}<0.05)$. The relative expression levels of JAK1 $(0.696 \pm 0.102)$ and STAT1 mRNA $(0.341 \pm 0.068)$ in the tumor tissue were lower than those in the benign tissue $(0.957 \pm 0.103$ and $0.547 \pm 0.082$, respectively; $\mathrm{P}<0.05)$. IFN stimulation enhanced the expression levels of P-STAT1 in the renal cell carcinoma tissues, and enhancement of the P-STAT1 expression levels was associated with tumor relapse and metastasis. In conclusion, P-STAT1 is a potential predictor of IFN response in patients with advanced renal cell carcinoma.
\end{abstract}

\section{Introduction}

Renal cell carcinoma is the most common type of malignant kidney tumor. It was estimated that in $2012 \sim 64,770$ new cases of kidney cancer would be diagnosed in the United States,

Correspondence to: Dr Wei Li, Department of Urology, The People's Hospital of Guangxi Zhuang Autonomous Region, 6 Taoyuan Road, Nanning, Guangxi 530021, P.R. China

E-mail: liwei95_2000@163.com

Key words: phosphorylated Signal Transducer and Activator of Transcription 1 , interferon- $\alpha$, renal cell carcinoma resulting in 13,570 mortalities (1). In 2011, 90\% of cases of kidney cancer were renal cell carcinoma (2). According to a review in $2008,30 \%$ of patients show signs of advanced renal cell carcinoma when diagnosed, and 20-30\% of patients treated by nephrectomy relapse and develop metastases during the follow-up period (3). As renal cell carcinoma is not sensitive to chemotherapy and radiotherapy, immunotherapy with interferon (IFN)- $\alpha$ was frequently used in the past 20 years (4). However, only a $15-30 \%$ objective response rate was reported was reported in a previous study and serious adverse effects have been observed in patients who have undergone immunotherapy (5). Thus, the ability to identify patients who are most likely to benefit from immunotherapy is necessary.

IFN- $\alpha$ exerts its effects on cells by interacting with the cell receptors and thus activating the Janus kinase (JAK) family. Activated JAK1 and tyrosine kinase 2 phosphorylate Signal Transducer and Activator of Transcription (STAT) and the activated STAT proteins interact with regulatory elements to induce the transcription of target genes (6). A previous study demonstrated that JAK-STAT activation is associated with IFN- $\alpha$ resistance in renal cell lines (7). Certain molecules, including STAT3 and p53, have been identified as predictive and prognostic markers of renal cell carcinoma $(8,9)$. However, the majority of these studies were conducted in vitro and these findings do not provide sufficient information to permit clinical treatment. To the best of our knowledge, studies concerning the expression of JAK-STAT in human renal cancer tissue are limited. The purpose of the present study was to assess the expression levels of JAK-STAT in human renal cancer tissues and identify a biomarker that is associated with the effects of IFN- $\alpha$ treatment.

\section{Patients and methods}

Patients and tissues. A total of 32 Chinese patients (mean age, 61.2 years) who had been diagnosed with locally advanced renal cell carcinoma by post surgery pathological examination at The People's Hospital of Guangxi Zhuang Autonomous Region (Nanning, China) between 2010 and 2011 were included in this study. The TNM staging of cancer was performed according to the American Joint Committee on Cancer Cancer Staging Manual (10). The demographic and clinicopathological charac- 
teristics of the patients are listed in Table I. A routine physical examination was conducted and the immune function of the patients was detected prior to the surgery. No abnormalities were identified in the patients. All patients received a radical nephrectomy and post surgery adjuvant immunotherapy with IFN- $\alpha 2 b$ (Schering-Plough, Kenilworth, NJ, USA). The treatment protocol was performed according to the instructions in the guidelines of the Chinese Urological Association (11). Considering the toxic side-effects of IFN, the patients were advised to receive a low dose of IFN- $\alpha 2 b$ (3 MIU subcutaneously, three times weekly) treatment for 12 weeks. The patients were followed-up for 24 months, and the local relapse and metastasis rates were recorded.

The tumor samples from the 32 patients were collected as soon as they were removed from the body. Each tumor sample was divided into two sections. One section was ground and stimulated in RPMI-1640 medium (HyClone Laboratories, Inc., Logan, UT, USA) containing IFN- $\alpha 2 b$ (final concentration, $3 \mathrm{MIU} / \mathrm{ml}$ ) for $4 \mathrm{~h}$, and the other section was incubated in RPMI-1640 medium without IFN, which served as a control. The benign renal tissues were collected from 10 patients who underwent a nephrectomy as a result of nonmalignant disease. All samples were stored at $-80^{\circ} \mathrm{C}$. The present study was approved by the Ethics Committee of The People's Hospital of Guangxi Zhuang Autonomous Region. Written consent was obtained from all patients.

Immunohistochemistry. The expression levels of JAK1, P-JAK1, STAT1,P-STAT1, STAT2 and P-STAT2 were detected by immunohistochemical staining of the renal cell carcinomas and benign renal tissues. The antibodies of P-JAK1, STAT2 and P-STAT2 were purchased from Abcam (Cambridge, UK). The antibodies of JAK1, STAT1 and P-STAT1 were purchased from Cell Signaling Technology, Inc. (Danvers, MA, USA). The immunohistochemical staining and evaluation were performed as previously described (12). All sections were blindly analyzed by experienced pathologists. Briefly, the immunohistochemical analysis was performed after recoding the sections to create a blind set. The overall intensity and percentage were evaluated under a light microscope (Olympus, Tokyo, Japan). Briefly, the immunohistochemical results were categorized into four grades based on the percentage of positive cells and/or the staining intensity, which was identified by comparing the section with the positive controls in each experiment. The grades were as follows: (-), positive cells $<5 \%$ of the cancer tissue and/or weakly stained; $(+),<25 \%$ and/or weakly stained; $(++),<50 \%$ and/or moderately stained; and $(+++),>75 \%$ and/or strongly stained.

Semiquantitative polymerase chain reaction ( $q P C R$ ) assay. Total RNA was isolated from the tumor and benign tissues using TRIzol ${ }^{\circledR}$ reagent (Invitrogen, Carlsbad, CA, USA). The RNA concentration was determined using a spectrophotometer (Bio-Rad Laboratories, Hercules, CA, USA). Complementary DNA was synthesized from $3 \mu \mathrm{g}$ total RNA with a RevertAid First Strand cDNA Synthesis kit (Fermentas, Hanover, MD, USA) according to the manufacturer's instructions. The qPCR assay was performed with $20 \mu \mathrm{l}$ reaction mixture, and the PCR was conducted with 35 cycles of $94^{\circ} \mathrm{C}$ for $30 \mathrm{sec}$ and $72^{\circ} \mathrm{C}$ for $1 \mathrm{~min}$. $\beta$-actin served as an internal
Table I. Demographic and clinicopathological characteristics of the patients.

\begin{tabular}{lr}
\hline Characteristic & Number \\
\hline Age, years & \\
$<50$ & 5 \\
$50-70$ & 20 \\
$>70$ & 7 \\
Gender & \\
Male & 22 \\
Female & 10 \\
$\mathrm{TNM}_{\mathrm{N}}$ stage & \\
$\mathrm{T}_{2} \mathrm{~N}_{1} \mathrm{M}_{0}$ & 16 \\
$\mathrm{~T}_{3 \mathrm{a}} \mathrm{N}_{0} \mathrm{M}_{0}$ & 11 \\
$\mathrm{~T}_{3 \mathrm{a}} \mathrm{N}_{1} \mathrm{M}_{0}$ & 3 \\
$\mathrm{~T}_{3 \mathrm{~b}} \mathrm{~N}_{0} \mathrm{M}_{0}$ & 2 \\
\hline
\end{tabular}

TNM, tumor node metastasis.

control. The PCR products underwent electrophoresis by $2 \%$ agarose gel analysis. The expression levels of the target genes were evaluated by calculating the average ratios of gray scale using a computerized gel imaging system (Bio-Rad Laboratories). The primers that were used for the amplification are listed in Table II.

Western blot analysis. The tumor samples, which had been stimulated with IFN, were lysed and homogenized (SanShon Machinery, Zhejiang, China) in radioimmunoprecipitation assay buffer and the control samples were treated in the same way. The protein concentration in the samples was subsequently determined. Equal amounts of protein were separated on $10 \%$ sodium dodecyl sulfate-polyacrylamide gel electrophoresis gels and transferred to a polyvinylidene difluoride membrane (Millipore, Billerica, MA, USA). The membrane was blocked with non-fat milk for $2 \mathrm{~h}$ and incubated with the primary antibodies overnight. Following washing with Tris-buffered saline and Tween 20 (TBST) for $30 \mathrm{~min}$, the membrane was incubated for $1 \mathrm{~h}$, washed with TBST and the proteins were detected with an enhanced chemiluminescence kit (Bio-Rad Laboratories). The protein expression levels were evaluated by calculating the average ratios of gray scale using a computerized gel imaging system; GAPDH served as the control.

Statistical analysis. The positive expression rates between the tumor and benign tissues were assessed with $\chi^{2}$ test. The qPCR and western blot data were analyzed using Student's t-test. $\mathrm{P}<0.05$ was considered to indicate a statistically significant difference. The data were analyzed with SPSS software version 19.0 (IBM, Armonk, NY, USA).

\section{Results}

Immunohistochemical staining in the renal cell carcinomas and benign renal tissues. Positive immunohistochemical 
Table II. Sequences of the primers used.

\begin{tabular}{llcr}
\hline Gene & \multicolumn{1}{c}{ Sequence $\left(5^{\prime} \rightarrow 3^{\prime}\right)$} & Length $(\mathrm{bp})$ & $\mathrm{T}_{\mathrm{m}}\left({ }^{\circ} \mathrm{C}\right)$ \\
\hline JAK1 & Sense: TTCTACATGGGGGGATAG & 54.4 \\
& Antisense: TAAGTATGGAAACCCTCTAA & 278 & 57.4 \\
STAT1 & Sense: GGTTGAACCCTACACGAAG & 335 & 59.8 \\
STAT2 & Antisense: CTACAGAGCCCACTATCCG & 368 & \\
& Sense: ATACTAGGGACGGGAAGTCG & & \\
$\beta$-actin & Antisense: CTGGGAAAAGGGCTGAATG & 306 & \\
& Sense: CGTGGACATCCGCAAAGAC & & \\
& Antisense: AAGAAAGGGTGTAACGCAACT & & \\
\hline
\end{tabular}

JAK, Janus kinase; STAT, Signal Transducer and Activator of Transcription.

A

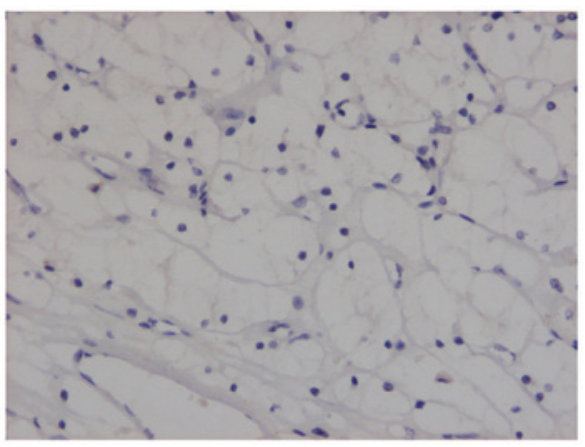

B

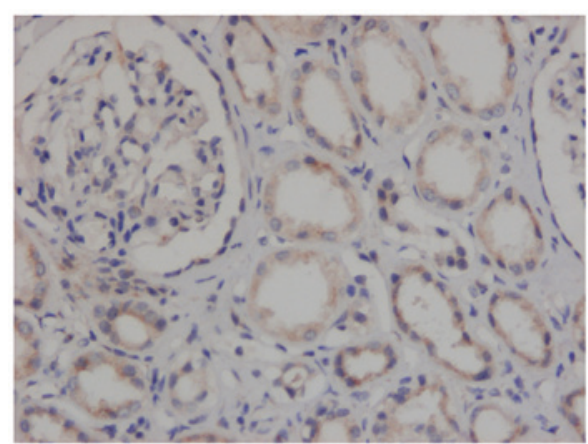

Figure 1. (A) Negative expression of Janus kinase 1 (JAK1) in renal cancer tissue. (B) Positive expression of JAK1 in benign renal tissue. Magnification, x200; staining, 3,3'-diaminobenzidine.
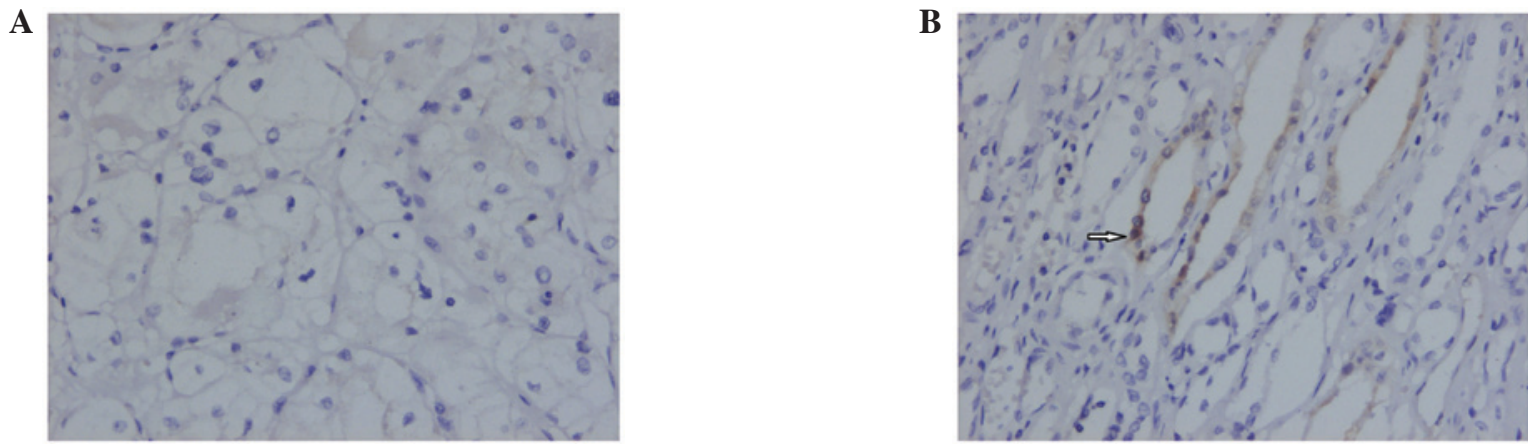

Figure 2. (A) Negative expression of Signal Transducer and Activator of Transcription 1 (STAT1) in renal cancer tissue. (B) Positive expression of STAT1 in benign renal tissue. The arrow indicates a positive stain in the cytoplasm of a distal convoluted tubule. Magnification, x200; staining, 3,3'-diaminobenzidine.

A

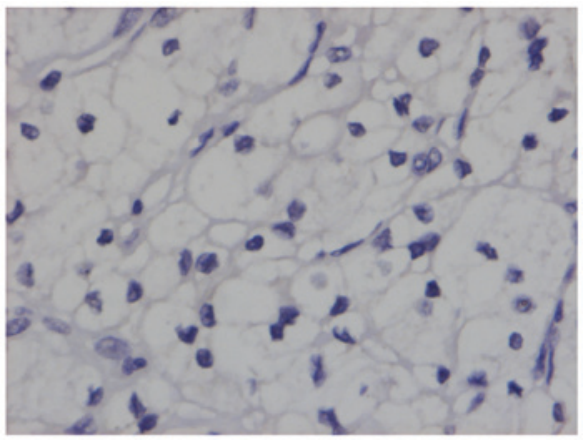

B

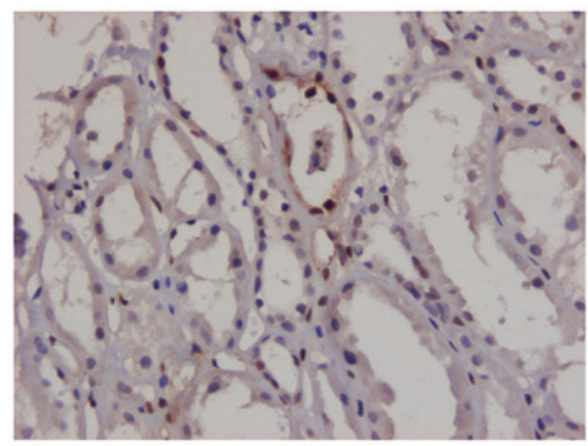

Figure 3. (A) Negative expression of phosphorylated Signal Transducer and Activator of Transcription 1 (P-STAT1) in renal cancer tissue. Magnification, x400. (B) Positive expression of P-STAT1 in benign renal tissue. Magnification, x200; staining, 3,3'-diaminobenzidine. 
Table III. Expression rates of JAK-STAT signaling pathway components in renal cell carcinoma and benign renal tissue.

\begin{tabular}{|c|c|c|c|c|}
\hline \multirow{2}{*}{$\begin{array}{l}\text { Signaling } \\
\text { pathway } \\
\text { component }\end{array}$} & \multicolumn{2}{|c|}{ Renal cell carcinoma, \% (n) } & \multicolumn{2}{|c|}{ Benign renal tissue, \% (n) } \\
\hline & - & + to +++ & - & + to +++ \\
\hline JAK1 & $75.0(24 / 32)$ & $25.0(8 / 32)$ & $30.0(3 / 10)$ & $70.0(7 / 10)$ \\
\hline P-JAK1 & $100.0(32 / 32)$ & $0.0(0 / 32)$ & $100.0(10 / 10)$ & $0.0(0 / 10)$ \\
\hline STAT1 & $68.8(22 / 32)$ & $31.2(10 / 32)$ & $50.0(5 / 10)$ & $50.0(5 / 10)$ \\
\hline P-STAT1 & $87.5(28 / 32)$ & $12.5(4 / 32)$ & $30.0(3 / 10)$ & $70.0(7 / 10)$ \\
\hline STAT2 & $0.0(0 / 32)$ & $100.0(32 / 32)$ & $20.0(2 / 10)$ & $80.0(8 / 10)$ \\
\hline P-STAT2 & $0.0(0 / 32)$ & $100.0(32 / 32)$ & $0.0(0 / 10)$ & $100.0(10 / 10)$ \\
\hline
\end{tabular}

JAK, Janus kinase; P, phosphorylated; STAT, Signal Transducer and Activator of Transcription.

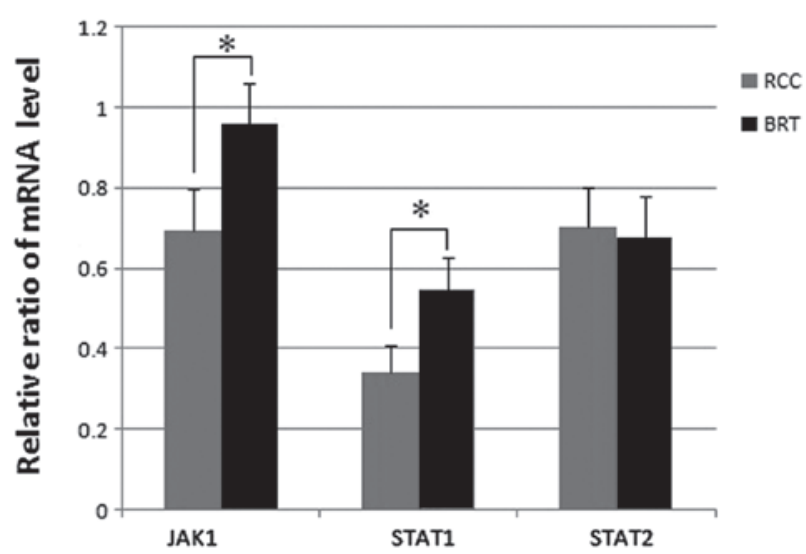

Figure 4. Relative ratio of the mRNA levels of JAK-STAT ( $\mathrm{P}<0.05)$. JAK, Janus kinase; STAT, Signal Transducer and Activator of Transcription; RCC, renal cell carcinomas; BRT, benign renal tissues.

staining was observed in the cytoplasm or cytomembranes of the renal tubules. The positive expression rates of JAK1, STAT1 and P-STAT1 in the renal cell carcinomas were significantly lower than those observed in the benign renal tissues $(\mathrm{P}<0.05)$. The expression rates of STAT2 in the renal cell carcinomas were elevated compared with those in the benign renal tissues $(\mathrm{P}<0.05)$. No differences in the expression rates of P-JAK1 and P-STAT2 between the renal cell carcinoma tissues and benign renal tissues were identified (Figs. 1-3; Table III).

qPCR detection of the JAK-STAT mRNA expression levels. The JAK1, STAT1 and STAT2 mRNA levels were detected in the renal cell carcinomas and benign renal tissues. The relative expression levels of JAK1 $(0.696 \pm 0.102)$ and STAT1 $(0.341 \pm 0.068)$ in the tumor tissues were lower than those in the benign tissues $(0.957 \pm 0.103$ and $0.547 \pm 0.082$, respectively; $\mathrm{P}<0.05)$. No significant differences were identified in the relative mRNA expression levels of STAT2 $(0.702 \pm 0.101$ vs. $0.676 \pm 0.105$ ) between the two types of tissue (Fig. 4).

Western blot analysis determined the changes in the levels of JAK-STAT expression following stimulation with IFN. To determine the active status of JAK-STAT in the tumor tissues

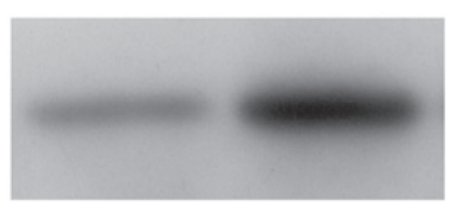

Figure 5. High enhanced expression levels of phosphorylated Signal Transducer and Activator of Transcription 1 (91 kDa). Right band: Stimulated with interferon- $\alpha 2 b$.

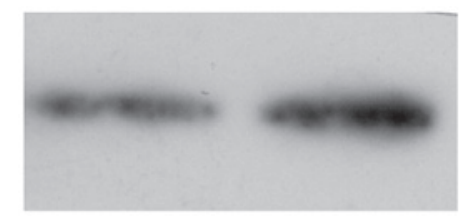

Figure 6. Low enhanced expression levels of phosphorylated Signal Transducer and Activator of Transcription 1 (91 kDa). Right band: Stimulated with interferon- $\alpha 2 b$.

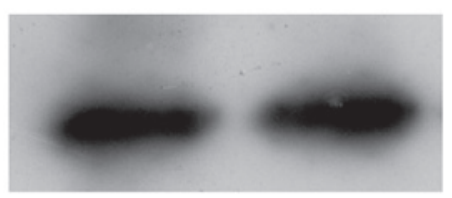

Figure 7. GAPDH (36 kDa).

following stimulation with IFN- $\alpha 2 b$, the expression levels of JAK-STAT in the IFN stimulated and control tumor tissues were identified using western blot analysis. The expression levels of JAK1, P-JAK1, STAT1 and P-STAT1 were examined in the two types of tissues. The results showed that IFN stimulation significantly increased the expression levels of P-STAT1 (Figs. 5-7), however, not those of STAT1, JAK1 and P-JAK1 $(\mathrm{P}<0.05$; Table IV). Enhanced expression levels of P-STAT1 were detected in $75 \%$ (24/32) of the samples, however, the levels varied markedly between the samples. Enhanced expression levels of P-STAT1 were not detected in $25 \%$ of the samples. The percentage of enhanced expression levels of P-STAT1 increased significantly when compared with those of the control $(\mathrm{P}<0.05)$. 
Table IV. Relative expression levels of JAK-STAT signaling pathway components prior to and following stimulation with interferon $(n=32)$.

Relative expression level, mean \pm standard deviation

Pathway components

Control tumor tissue

Stimulated tumor tissue

\begin{tabular}{llr}
\hline JAK1 & $0.253 \pm 0.14$ & $0.298 \pm 0.19$ \\
P-JAK1 & $0.316 \pm 0.23$ & $0.307 \pm 0.14$ \\
STAT1 & $0.424 \pm 0.27$ & $0.384 \pm 0.20$ \\
P-STAT1 & $0.190 \pm 0.12$ & $0.460 \pm 0.24^{\mathrm{a}}$
\end{tabular}

${ }^{a} \mathrm{P}<0.05$, compared with control tumor tissue. JAK, Janus kinase; P, phosphorylated; STAT, Signal Transducer and Activator of Transcription.

Table V. Local relapse and metastasis rates of the renal cell carcinomas in the two groups; high and low expression of phosphorylated Signal Transducer and Activator of Transcription.

\begin{tabular}{lcr}
\hline Group & Local relapse rate, \% (n) & Metastasis rate, \% (n) \\
\hline High expression & $16.7(2 / 12)$ & $0.0(0 / 10)$ \\
Low expression & $35.0(7 / 20)$ & $5.0(1 / 20)$ \\
\hline
\end{tabular}

Enhanced P-STAT1 expression levels were associated with relapse and metastasis of renal cell carcinoma. The patients were followed up for 24 months after the immunotherapy with IFN. Clinical evaluation was performed by regular interviews, and relapse and metastasis of the tumors were diagnosed using enhanced computed tomography scanning and tissue biopsy analysis. The patients were divided into two groups (high and low expression) based on whether the enhancement of the P-STAT1 expression levels was above or below the mean value. The rates of relapse and metastasis were compared between the high and low expression level groups. All patients tolerated the immunotherapy well and completed their treatment courses. By the end of the follow-up period, nine patients suffered local relapse and lung metastasis was identified in one patient. The high expression levels group exhibited reduced relapse and metastatic rates compared with those of the low expression group $(\mathrm{P}<0.05$; Table $\mathrm{V})$.

\section{Discussion}

Although IFN- $\alpha$ is a treatment option for advanced and metastatic renal cell carcinoma, a substantial proportion of patients fail to respond to IFN treatment (13). The mechanism of resistance to IFN treatment of renal cell carcinoma remains unclear. The cytokine-activated JAK-STAT pathway has an important role in the control of immune responses. The present study focused on the expression status of the components of the JAK-STAT pathway in human renal cell carcinoma and attempted to identify a biomarker, which predicts the IFN response in patients undergoing immunotherapy.

The expression levels of JAK-STAT in renal cancer carcinoma tissues were examined and the results indicated that the positive expression rates of JAK1, STAT1 and P-STAT1 in the renal cell carcinomas were significantly lower compared with those in the benign renal tissues. Furthermore, the
qPCR assay showed that the relative mRNA levels of JAK1 and STAT1 in the malignant tissues were lower than those in the benign tissues. A previous study on IFN-resistant human melanoma cells demonstrated that a defect in the levels of STAT1 was responsible for the reduced response to IFN treatment, and transfection of the IFN-resistant cell line to express increased levels of STAT1 partially restored the IFN response (14). In addition, the results from an in vitro study indicated that an IFN-resistant renal carcinoma cell line was associated with defective induction of STAT1 (15). The results of the present study demonstrated that defective expression of the JAK-STAT1 pathway may be a common phenomenon in IFN-resistant malignant tumors. Furthermore, the results of defective expression levels of JAK1 and STAT1 in human renal carcinoma tissues, which were observed in the present study, were consistent with the findings from a previous cell line study (7).

The change in the expression levels of JAK, P-JAK1, STAT1 and P-STAT1 following IFN stimulation was investigated in the present study using immunohistochemical staining and qPCR. IFNs are able to bind to a specific cell surface IFN receptor, and IFN receptor activation leads to the phosphorylation of STAT1 and STAT2, which is critical for signal transduction (16). The results of the present study showed that IFN stimulation increased the expression levels of P-STAT1. Enhanced expression levels were detected in $75 \%$ (24/32) of the tumor samples. These results indicated that the majority of tumors respond to IFN stimulation, however, the response in the present study varied markedly between the tumor samples, which may indicate different responses to IFN therapy among patients. As only the enhanced expression levels of P-STAT1 were detected by western blot analysis, the association between the enhanced expression levels of P-STAT1 and the response to IFN therapy in patients was subsequently investigated. All patients who had undergone IFN therapy for 24 months were followed up and 
the results indicated that the patients whose tumors expressed P-STAT1 in high levels following IFN stimulation were less likely to suffer a relapse or metastasis. In addition, the post surgery recurrence rate of locally advanced renal cell carcinoma was $64 \%$ according to the data of a previous study (17), however, the rate of relapse in the high expression levels group in the present study was $16.7 \%$, which indicated that the lower relapse rate in the present study was due to the IFN therapy.

In conclusion, numerous attempts have been made to identify a predictive biomarker for IFN resistance in different fields $(8,18,19)$. The results of the present study indicated that P-STAT1 may be a novel predictor of the IFN response in patients with advanced renal cell carcinoma. This study presents the preliminary results, however, a greater population of patients and an increased follow-up period are required for further investigation.

\section{Acknowledgements}

The present study was funded by the Natural Science Foundation of Guangxi, China (grant no. 2010GXNSFB013080).

\section{References}

1. Siegel R, Naishadham D and Jemal A: Cancer statistics, 2012. CA Cancer J Clin 62: 10-29, 2012.

2. Ljungberg B, Campbell SC, Choi HY, Jacqmin D, Lee JE, Weikert S and Kiemeney LA: The epidemiology of renal cell carcinoma. Eur Urol 60: 615-621, 2011.

3. Athar $U$ and Gentile TC: Treatment options for metastatic renal cell carcinoma: a review. Can J Urol 15: 3954-3966, 2008.

4. Jurado JM, Zarcos I, Delgado M, Blancas I, Legerén M and García-Puche JL: Temsirolimus in overtreated metastatic renal cancer with subsequent use of sunitinib: A case report. Oncol Lett 5: 1382-1384, 2013.

5. Tanriverdi O: Review on targeted treatment of patients with advanced-stage renal cell carcinoma: a medical oncologist's perspective. Asian Pac J Cancer Prev 14: 609-617, 2013.

6. Darnell JE Jr, Kerr IM and Stark GR: Jak-STAT pathways and transcriptional activation in response to IFNs and other extracellular signaling proteins. Science 264: 1415-1421, 1994.

7. Shang D, Liu Y, Ito N, Kamoto T and Ogawa O: Defective Jak-Stat activation in renal cell carcinoma is associated with interferon-alpha resistance. Cancer Sci 98: 1259-1264, 2007.
8. Eto M, Kamba T, Miyake H, Fujisawa M, Kamai T, Uemura H, Tsukamoto T, et al; Japan Immunotherapy SNPs-Study Group for Kidney Cancer: STAT3 polymorphism can predict the response to interferon- $\alpha$ therapy in patients with metastatic renal cell carcinoma. Eur Urol 63: 745-752, 2013.

9. Masuda A, Kamai T, Abe H, Arai K and Yoshida K: Is Stat3 and/or p53 mRNA expression a prognostic marker for renal cell carcinoma? Biomed Res 30: 171-176, 2009.

10. Edge S, Byrd DR, Compton CC, Fritz AG, Greene FL and Trotti A (eds): AJCC Cancer Staging Manual. 7th ed. Springer Verlag, New York, NY, pp547-560, 2009.

11. Yanqun Na, Zhangqun Ye and Guang Sun: The CUA Guideline for Urological Disease. People's Health Publishing House, Beijing pp4-16, 2011 (In Chinese).

12. Chen L, Zhu YY, Zhang XJ, Wang GL, Li XY, He S, Zhang JB and Zhu JW: TSPAN1 protein expression: a significant prognostic indicator for patients with colorectal adenocarcinoma. World J Gastroenterol 15: 2270-2276, 2009.

13. Zeng Z, Que T, Zhang J and Hu Y: A study exploring critical pathways in clear cell renal cell carcinoma. Exp Ther Med 7: 121-130, 2014.

14. Wong LH, Krauer KG, Hatzinisiriou I, Estcourt MJ, Hersey P, Tam ND, Edmondson S, et al: Interferon-resistant human melanoma cells are deficient in ISGF3 components, STAT1, STAT2, and p48-ISGF3gamma. J Biol Chem 272: 28779-28785, 1997.

15. Brinckmann A, Axer S, Jakschies D, Dallmann I, Grosse J, Patzelt T, Bernier T, et al: Interferon-alpha resistance in renal carcinoma cells is associated with defective induction of signal transducer and activator of transcription 1 which can be restored by a supernatant of phorbol 12-myristate 13-acetate stimulated peripheral blood mononuclear cells. Br J Cancer 86: 449-455, 2002.

16. Caraglia M, Marra M, Pelaia G, Maselli R, Caputi M, Marsico SA and Abbruzzese A: Alpha-interferon and its effects on signal transduction pathways. J Cell Physiol 202: 323-335, 2005.

17. Lam JS, Shvarts O, Leppert JT, Pantuck AJ, Figlin RA and Belldegrun AS: Postoperative surveillance protocol for patients with localized and locally advanced renal cell carcinoma based on a validated prognostic nomogram and risk group stratification system. J Urol 174: 466-472, 2005.

18. Noguchi Y, Kurokawa MS, Okuse C, Matsumoto N, Nagai K, Sato T, Arito M, et al: Serum peptides, represented by complement $3 \mathrm{f}$ des-arginine, are useful for prediction of the response to pegylated interferon- $\alpha$ plus ribavirin in patients with chronic hepatitis C. Hepatol Res 43: 743-756, 2013.

19. Azuma T, Matayoshi Y, Nagase Y and Oshi M: Neutrophil number after interferon-alfa treatment is an independent predictive marker of overall survival in metastatic renal cell carcinoma. Clin Genitourin Cancer 10: 180-184, 2012. 